\title{
Long Pulse EBW Start-up Experiments in MAST
}

\author{
V.F. Shevchenko, a, Y.F. Baranov ${ }^{1}$, T. Bigelow ${ }^{2}$, J.B. Caughman ${ }^{2}$, S. Diem ${ }^{2}$, C. Dukes ${ }^{2}$, P. Finburg ${ }^{1}$, J. Hawes ${ }^{1}$, C. Gurl ${ }^{1}$, \\ J.Griffiths ${ }^{1}$, J. Mailloux ${ }^{1}$, M. Peng ${ }^{2}$, A.N. Saveliev ${ }^{3}$, Y. Takase ${ }^{4}$, H. Tanaka ${ }^{5}$, G. Taylor $^{6}$ \\ ${ }^{1}$ CCFE, Culham Science Centre, Abingdon, OX14 3DB, UK \\ ${ }^{2}$ Oak Ridge National Laboratory, Oak Ridge, Tennessee 37830, USA \\ ${ }^{3}$ Ioffe Institute, Politekhnicheskaya 26, 194021 St. Petersburg, Russia \\ ${ }^{4}$ University of Tokyo, Kashiwa 277-8561, Japan \\ ${ }^{5}$ Graduate School of Energy Science, Kyoto University, Kyoto 606-8502, Japan \\ ${ }^{6}$ Princeton Plasma Physics Laboratory, Princeton, New Jersey 08543, USA
}

\begin{abstract}
Start-up technique reported here relies on a double mode conversion (MC) for electron Bernstein wave (EBW) excitation. It consists of MC of the ordinary $(\mathrm{O})$ mode, entering the plasma from the low field side of the tokamak, into the extraordinary $(\mathrm{X})$ mode at a mirror-polarizer located at the high field side. The $\mathrm{X}$ mode propagates back to the plasma, passes through electron cyclotron resonance (ECR) and experiences a subsequent $\mathrm{X}$ to EBW MC near the upper hybrid resonance (UHR). Finally the excited EBW mode is totally absorbed at the Doppler shifted ECR. The absorption of EBW remains high even in cold rarefied plasmas. Furthermore, EBW can generate significant plasma current giving the prospect of a fully solenoid-free plasma start-up. First experiments using this scheme were carried out on MAST [1]. Plasma currents up to $33 \mathrm{kA}$ have been achieved using $28 \mathrm{GHz} 100 \mathrm{~kW} 90 \mathrm{~ms}$ RF pulses. Recently experimental results were extended to longer RF pulses showing further increase of plasma currents generated by RF power alone. A record current of $73 \mathrm{kA}$ has been achieved with $450 \mathrm{~ms}$ RF pulse of similar power. The current drive enhancement was mainly achieved due to RF pulse extension and further optimisation of the start-up scenario.
\end{abstract}

\section{Introduction}

Non-inductive plasma current start-up is a critical issue in spherical tokamak (ST) research because of lack of space for a shielded central solenoid. Various techniques have been proposed and developed in order to avoid a central solenoid in future ST devices [2-5]. In this paper, we report on the electron Bernstein wave (EBW) start-up technique based on a $28 \mathrm{GHz}$ gyrotron capable of delivering $300 \mathrm{~kW}$ power for up to $0.5 \mathrm{~s}$. Experiments were carried out on the Mega Amp Spherical Tokamak (MAST) based at Culham Science Centre, UK [6]. MAST has 5 pairs of poloidal field coils inside the vacuum vessel as shown in fig. 1. Upper and lower parts of P2 - P5 coils carry current in the same direction and generate vertical magnetic field required for plasma shaping and equilibrium. Upper and lower parts of the P6 coils carry current in opposite directions and generate radial magnetic field providing vertical control of the plasma. This set of poloidal coils except for P3 was used in the start-up experiments described below. The central rod $(\mathrm{CR})$ current generating toroidal magnetic field $\mathrm{B}_{\mathrm{T}}$ was set to be of 2 MA during these experiments.

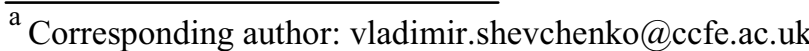



Figure 1. Poloidal field coils location in MAST (left) and a typical plasma cross-section (right). 
That gives a radial position of an electron cyclotron (EC) resonance $\left(\mathrm{B}_{\mathrm{T}}=1 \mathrm{~T}\right)$ at $0.4 \mathrm{~m}$. The method deployed here has been proposed in [7, 8] and first experiments were described in [1]. It relies on generation of low-density plasma by RF pre-ionization around the fundamental electron cyclotron resonance (ECR) with an ordinary $(\mathrm{O})$ mode, incident from the low field side of the tokamak. Most of the O-mode power passes through the plasma and hits CR. Then the power is reflected back by a grooved mirror-polarizer incorporated in a graphite tile on CR. Polarization of the reflected beam is rotated by $90^{\circ}$ to match an extraordinary (X) mode. The launched Gaussian beam is tilted to the midplane at $10^{\circ}$ and hits the $\mathrm{CR}$ at the midplane as illustrated in fig. 2. The Omode beam has an effective (electric field e-fold length) radius of about $80 \mathrm{~mm}$ which is well within the grooved area of $250 \times 250 \mathrm{~mm}^{2}$ on the CR.

The $\mathrm{X}$ mode reflected from the polarizer propagates back to the plasma, passes through ECR and experiences a subsequent slow $\mathrm{X}$ to EBW mode conversion (MC) near the upper hybrid resonance (UHR). Finally the excited EBW mode is totally absorbed before it reaches the ECR, due to the Doppler shifted resonance. Modelling shows that only a small fraction of the injected power $(\sim 2 \%)$ is absorbed as the $\mathrm{O}$ and $\mathrm{X}$ modes, while the main part is converted into the EBW mode. The absorption of EBW remains high even in cold rarefied plasmas. Furthermore, EBW can generate significant plasma current (if absorption is above or below the midplane) [9] during the start-up phase giving the prospect of a fully non-inductive plasma start-up.

\section{Long pulse experiments}

The original hardware was significantly upgraded since first experiments [1] in collaboration with Oak Ridge National Laboratory, USA. A short pulse gyrotron has been replaced with a $28 \mathrm{GHz} 200 \mathrm{~kW} \mathrm{CW}$ tube which is capable to deliver up to $300 \mathrm{~kW}$ in a pulsed mode with pulse duration up to $0.5 \mathrm{~s}$.

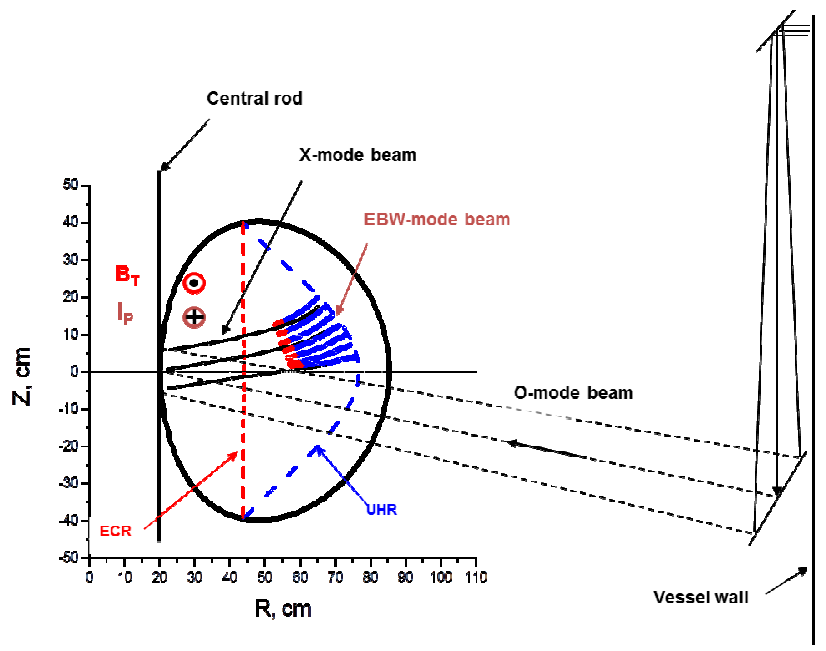

Figure 2. EBW assisted plasma start-up schematic. Red colour at the end of EBW rays indicates the power deposition zone.
An original transmission line based on smooth circular $\mathrm{TE}_{01}$ waveguides has been upgraded with larger diameter $\mathrm{HE}_{11}$ corrugated waveguides resulting in improvement of transmission efficiency by more than $25 \%$. However RF power injected into the MAST vessel was limited by arcing at the focusing optics near the vacuum window. So experiments reported here extend previous results only in terms of pulse duration with about the same level of RF power.

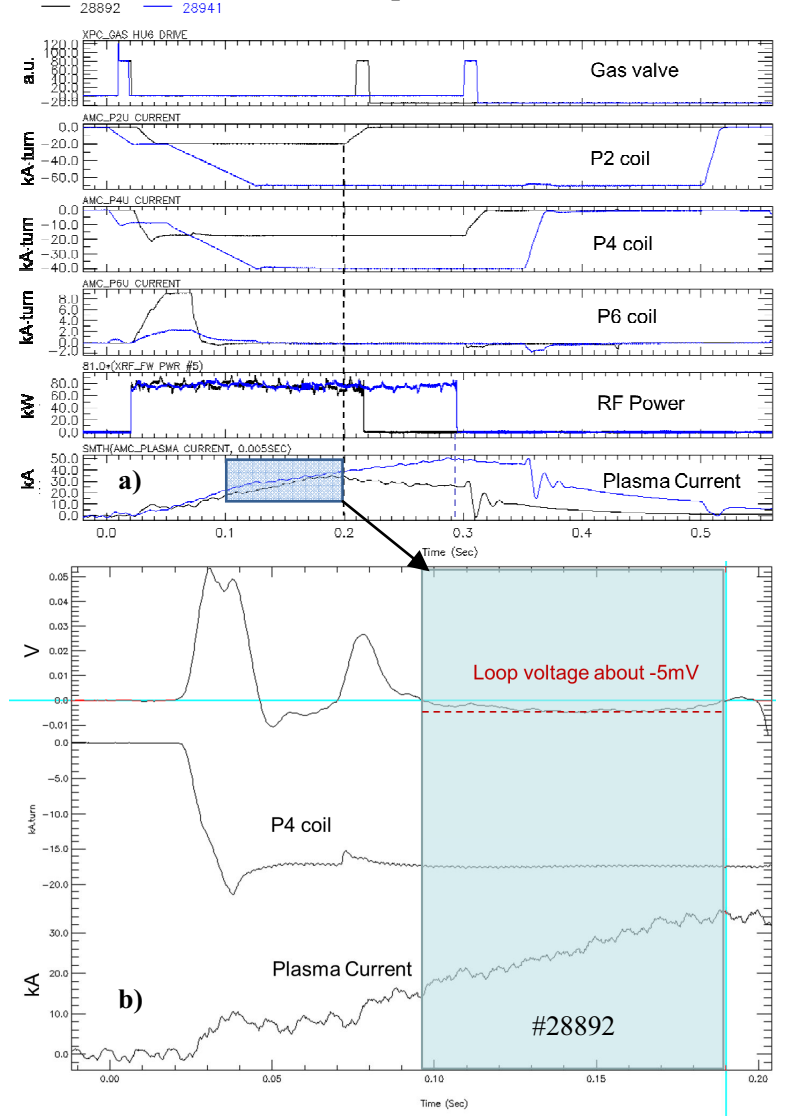

Figure 3. EBW start-up experiments in constant Bv: a) experimental waveforms with different pulse duration; b) zoomed-in waveforms illustrating negative loop voltage.

First let us consider EBW start-up in a constant vertical field $\mathrm{Bv}$. Waveforms are shown in fig. 3. A gas valve puffs the gas just before the RF pulse. In the shot \#28892 vertical field is produced by $\mathrm{P} 2$ and P4 coils and it reaches its stationary value of about $5 \mathrm{mT}$ at the midplane during $15 \mathrm{~ms}$ after RF onset. P6 coils produce a vertical kick to the plasma, which doesn't generate any loop voltage or pre-ionisation but helps to form closed flux surfaces (CFS) [1]. Plasma current monotonically increases from $0.1 \mathrm{~s}$ until $0.2 \mathrm{~s}$ when $\mathrm{P} 2$ current is switched off. After that it shows gradual decrease and it goes down with a faster rate after $\mathrm{P} 4$ switching off.

A negative loop voltage up to $-5 \mathrm{mV}$ is clearly observed in fig. $3 \mathrm{~b}$ during Bv stationary phase $0.1-0.2 \mathrm{~s}$. It achieves its most negative value at $0.15 \mathrm{~s}$ and then goes to zero at $0.19 \mathrm{~s}$. There are no other sources of toroidal electric field apart from increasing plasma current driven by RF power. Plasma current reaches its saturation at $0.19 \mathrm{~s}$ because $\mathrm{Bv}$ is not sufficient to confine the plasma from expansion. In the shot \#28941 Bv is partly set before the RF pulse. Then $\mathrm{Bv}$ is ramped up to a higher 
value during P6 vertical kick. Then all the fields remain constant from $0.12 \mathrm{~s}$ through the whole duration of the RF pulse until $0.29 \mathrm{~s}$. As in the previous shot plasma current monotonically increases until the end of RF injection. In both shots plasma current reaches the same value at $0.19 \mathrm{~s}$ but in the second one it is achieved with the smaller vertical kick but the higher Bv ramp-up. Monotonic growth of the plasma current until the end of RF pulse indicates that saturation has not been achieved with this Bv value. Fig. 4 illustrates different phases of plasma evolution during start-up in constant Bv. The first picture (fig. 4a) was taken at $0.15 \mathrm{~s}$ straight after CFS formation and the second one (fig. 4b) at $0.28 \mathrm{~s}$ close to end of the RF pulse when plasma has expanded both vertically and radially.
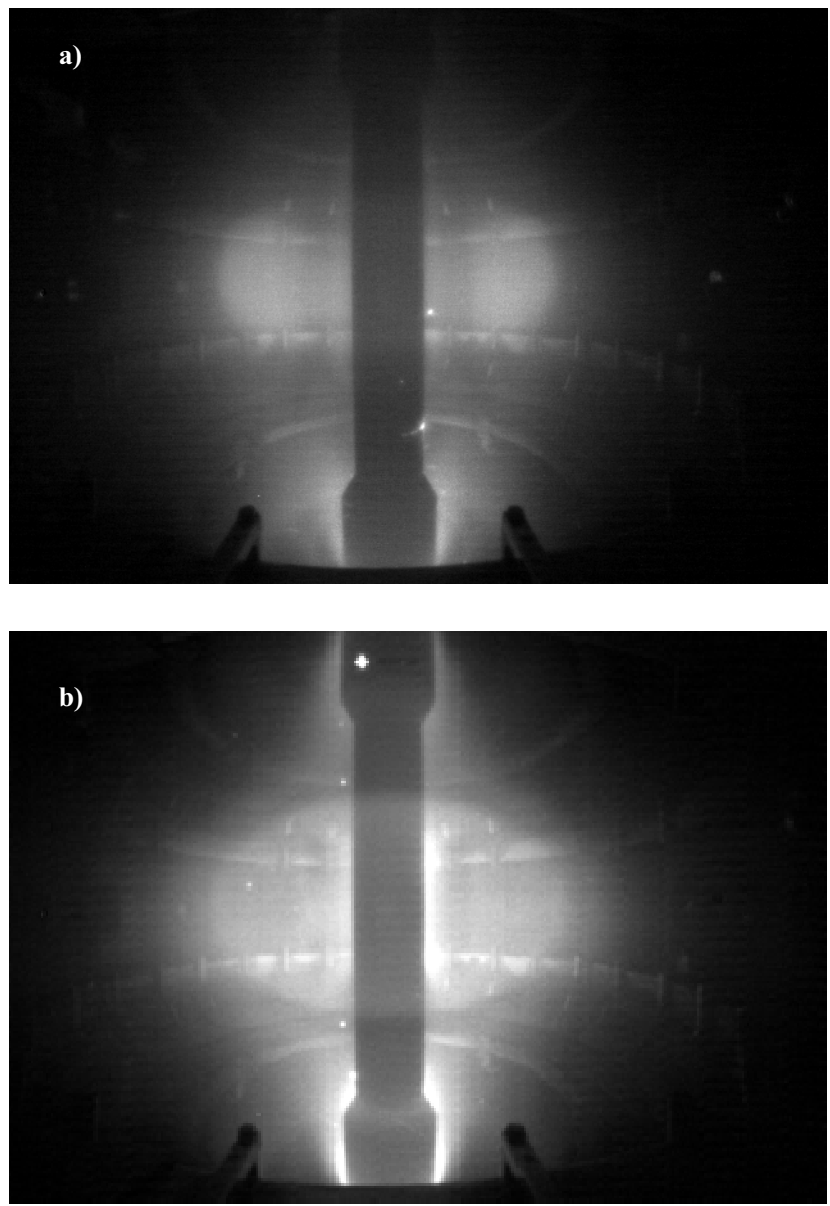

Figure 4. Plasma images taken during EBW start-up with the constant Bv \#28941: a) short after CFS formation; b) at the end of RF pulse.

It was shown experimentally that the most convenient way to achieve higher plasma current and keep plasma in equilibrium is to apply an optimised Bv ramp-up which holds the loop voltage close to zero until the end of a current ramp-up phase. Waveforms of plasma current generated by RF pulses of different duration in the presence the Bv ramp-up are shown in fig. 5. Here RF power of the same level was injected into the plasma for $320 \mathrm{~ms}, 400 \mathrm{~ms}$ and $440 \mathrm{~ms}$. It is clear from this figure that plasma currents drops down by $\sim 20 \%$ within $\sim 30 \mathrm{~ms}$ after the end of the RF pulse and remains almost constant until the end of the Bv ramp-up.



Figure 5. Effect of the RF pulse length with the ramping-up Bv.

Plasma density in these experiments was about $10^{18}$ $\mathrm{m}^{-3}$ and electron temperature within the range of 100-200 $\mathrm{eV}$ as measured by Thomson scattering. It was concluded in [1] that plasma current is predominantly carried by supra thermal electrons with the energy of 70-100 keV. Such electrons must be almost collisionless at the plasma density of $10^{18} \mathrm{~m}^{-3}$ so they should not be affected by the switch-off of the RF drive. Trapped electrons possess much lower energy than passing so the current carried by trapped electrons [1] can probably degrade within $30 \mathrm{~ms}$ providing the observed decrease of the total plasma current. The supra-thermal part of the current remains constant due to a small loop voltage generated by $\mathrm{Bv}$ ramp-up.

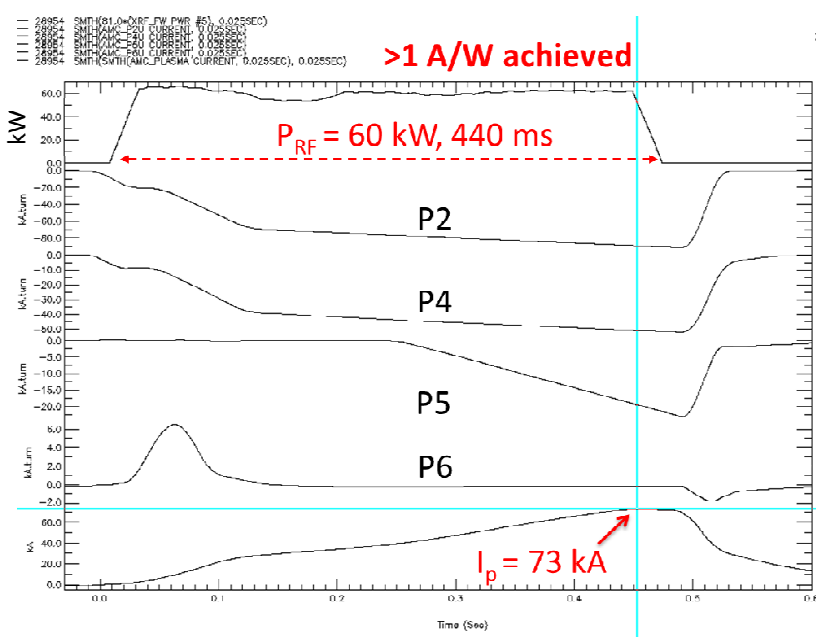

Figure 6. Waveforms of the shot with record plasma current achieved in EBW start-up.

A record current of $73 \mathrm{kA}$ has been achieved by optimisation of $\mathrm{Bv}$ waveforms as seen in fig. 6 . RF power of about $60 \mathrm{~kW}$ with pulse length of $0.44 \mathrm{~s}$ was injected into the MAST vessel. Three pairs of poloidal field coils were used to provide an optimised $\mathrm{Bv}$ shape and a rampup rate. Plasma current reached the value of $73 \mathrm{kA}$ at the end of the RF pulse and it would probably continue to rise further if $\mathrm{RF}$ power was available longer. Total efficiency of $1.2 \mathrm{~A} / \mathrm{W}$ has been achieved with this startup scenario. To our knowledge efficiencies close to 1 
A/W were previously reported only for start-up scenarios based on lower hybrid waves [10].

The plasma image taken at end of the RF pulse is shown in fig. 7 . The plasma has a clear toroidal structure with elongated poloidal cross-section. Unfortunately due to low density in this shot detailed Thomson scattering measurements of density and temperature profiles were not possible.

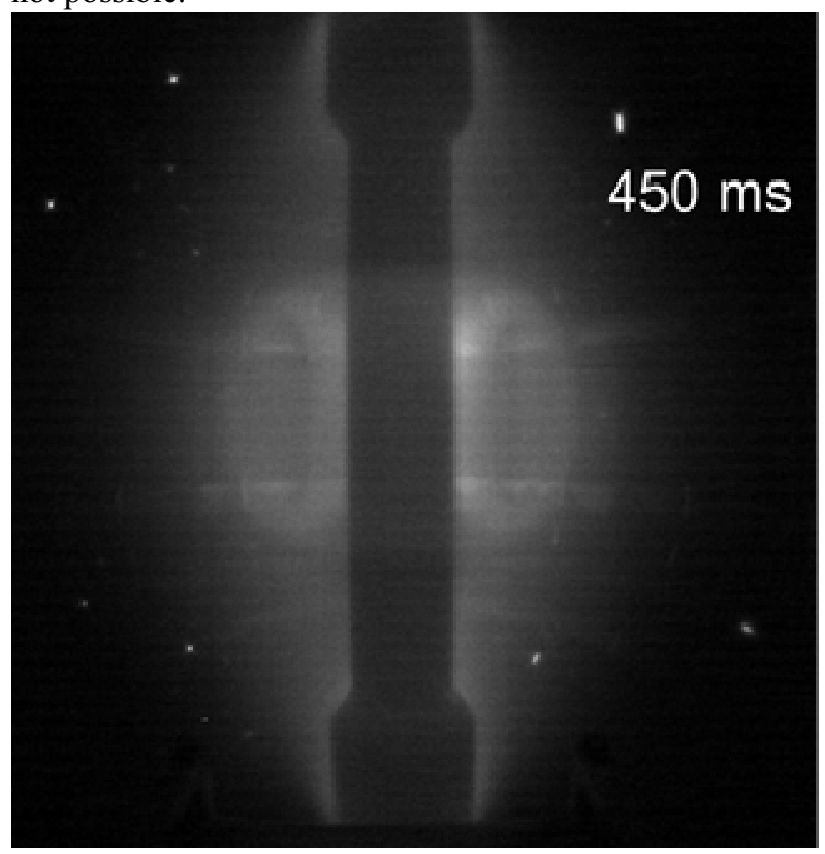

Figure 7. Plasma image at the end of RF pulse with the maximum plasma current of $73 \mathrm{kA}$.

As it was mentioned above experiments presented here were constrained by the arcing limiting RF power injected into MAST. However in relatively short pulses $(<0.2 \mathrm{~s})$ it was possible to inject RF power acceding 100 $\mathrm{kW}$. On the other end it was found experimentally that the lowest power level which is still capable to reproduce the above start-up scenario is about $40 \mathrm{~kW}$.

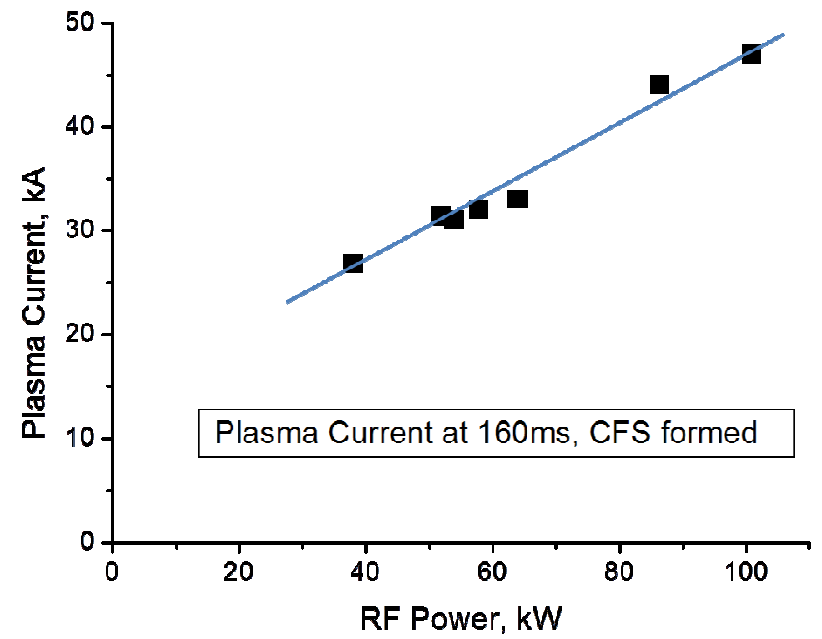

Figure 8. Scaling of the start-up current measured after CFS formation against injected RF power.

Due to the fact that the higher power shots were limited in duration we have chosen a time slice available for comparison in the majority of experiments. That is a moment when CFSs were formed and initial Bv ramp-up and P6 vertical kick were completed. For the majority of shots that happens after $150 \mathrm{~ms}$ so the time slice at 160 ms was chosen for comparison. The results are depicted in fig. 8 .

Interestingly all experimental points fit well into a linear dependence of generated plasma current versus RF power injected into the plasma. At least this dependence seems to be valid within the range of RF power available in the experiments reported in this paper. From FokkerPlanck modelling it was expected stronger than linear dependence of plasma current against power. Probably it can be observed at higher RF power levels.

\section{Experiments with vertical modulation}

It was predicted in [11] and experimentally confirmed in [9] that EBW power deposition location with respect to the plasma midplane is crucial for the EBW current drive (CD) direction.
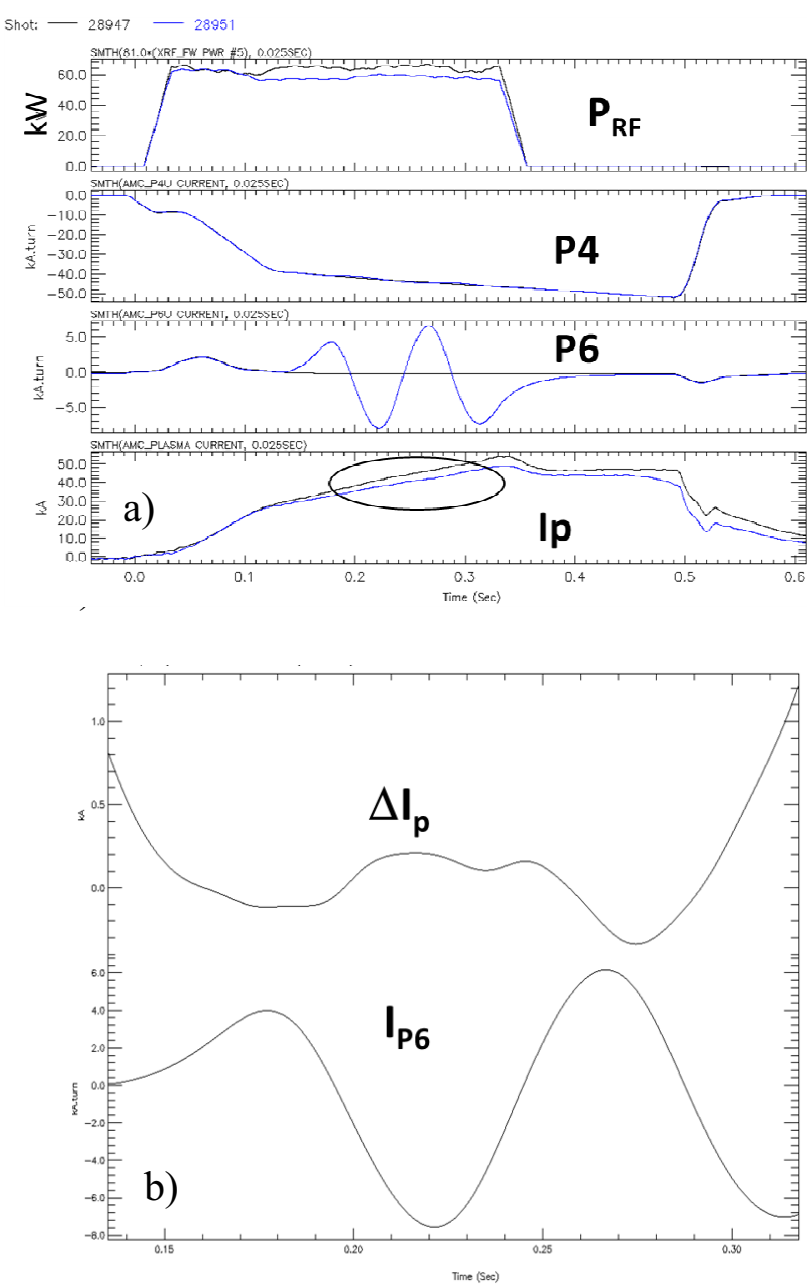

Figure 9. Waveforms of the experiment with plasma vertical modulation: a) reference and test shots; b) subtracted reference plasma current.

A special experiment was conducted to verify the CD mechanism responsible for the current generation in the start-up scenario under discussion. To change the EBW power deposition from the RF side is impossible in the 
MAST tokamak. The RF launching system was carefully pre-aligned to provide efficient $\mathrm{MC}$ on the mirrorpolariser which is fixed at the midplane on the CR. However it is possible to move the plasma midplane with respect to the machine midplane by applying radial magnetic fields produced by P6 coils.

The experiment was conducted as follow. A reference shot was produced by $60 \mathrm{~kW} 350 \mathrm{~ms}$ RF pulse with the same Bv waveforms as in fig. 6 apart from P5 which was disabled in these experiments. Then several test shots were fired with 2 period sinusoidal modulations applied to radial field coils P6 starting at $0.15 \mathrm{~s}$ as shown in fig. 9a. This sort of modulation produced vertical up and then down plasma shifts of about $+/-15 \mathrm{~cm}$ which were clearly visible on fast video records. Plasma current modulations induced by vertical shifts are barely visible on the Ip waveforms. However after a subtraction of the plasma current from the reference shot and an offset removal the effect of modulation becomes obvious. The plasma current decreases when the plasma moves up (positive $\mathrm{I}_{\mathrm{P} 6}$ ) and increases when the plasma moves down (negative $\mathrm{I}_{\mathrm{P} 6}$ ). That is exactly what was predicted by Fokker-Planck modelling.

Such behaviour can be understood from the following consideration. In a non-disturbed position shown in fig. 2 almost all EBW power is absorbed above the midplane. This power produces $\mathrm{CD}$ in a co-direction. When plasma moved up while the RF beam remained fixed some fraction of the EBW power will be deposited below the plasma midplane causing $\mathrm{CD}$ in a counterdirection. That will result in a reduction of the total CD effect hence a reduction in the plasma current. Inversely when plasma moves down the fraction of the power deposited above the plasma midplane becomes larger resulting in the increase of the plasma current. These experiments qualitatively prove the EBW CD mechanism in this start-up scenario. However quantitative agreement between modelling and experiments is not ideal. Modelling shows several times larger response of the plasma current. This disagreement may be partly explained by the assumption of the Maxwellian plasma in the ray-tracing and Fokker-Planck modelling which was clearly not the case. Appropriate corrections are under development.

\section{ECE from start-up plasma}

Another experiment which can help to understand the physics of EBW start-up was aimed to measure EC emission (ECE) from the plasma during the start-up phase. A radiometer was receiving horizontally polarised ECE from two viewing directions simultaneously. One viewing direction was close to perpendicular $\left(N_{\|} \sim 0\right)$ to the magnetic field and another one close to tangential $\left(N_{\|}\right.$ $\sim 1$ ) at the radius of $0.8 \mathrm{~m}$. The radiometer frequency coverage was from $24.5 \mathrm{GHz}$ to $29.5 \mathrm{GHz}$ with the gyrotron frequency of $28 \mathrm{GHz}$ filtered out by a notch filter. All measurements discussed below were obtained with a $10 \mathrm{~dB}$ attenuator installed at the antenna feed in comparison with a routine plasma operation where no attenuation is required. It indicates that ECE intensity during EBW start-up was at least an order of magnitude higher compared with thermal ECE from 'normal' plasma in MAST.
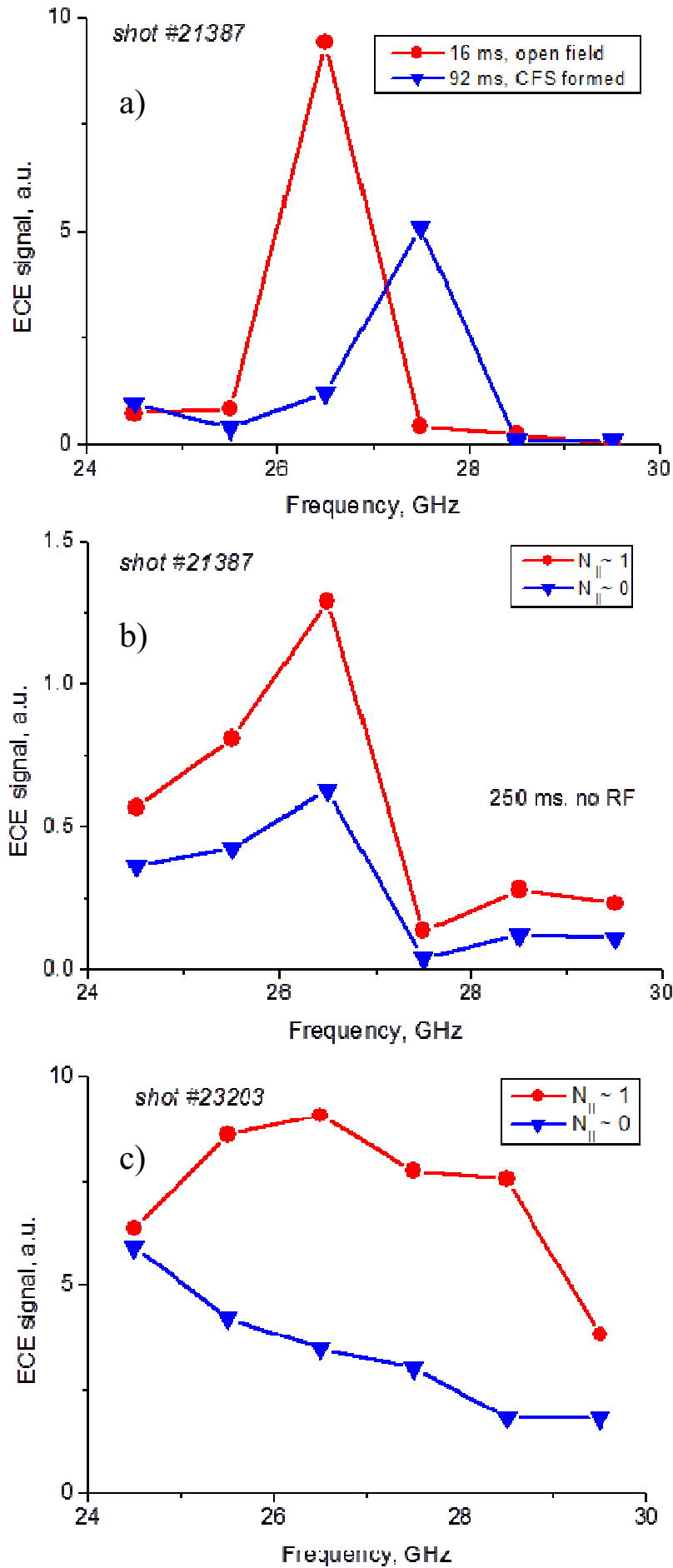

Figure 10. ECE spectra measured during EBW start-up: a) initial stage before CFS formation (red circles), shortly after CFS formation (blue triangles); b) after the end of RF pulse; c) in the presence of RF and a limited solenoid assist.

Results are summarised in fig. 10. The first fig. 10a shows ECE spectra both measured with $N_{\|} \sim 1$ during RF injection at the initial part of the start-up. The data shown in red circles was taken at $16^{\text {th }} \mathrm{ms}$ in the open field 
configuration when plasma rapidly expanded and plasma current was below $10 \mathrm{kA}$. Confinement at this stage is very poor so electrons re-emit ECE straight after they have been energised by RF. There is a clear maximum at $26.5 \mathrm{GHz}$ down-shifted form the gyrotron frequency by $1.5 \mathrm{GHz}$. From relativistic resonance condition the energy about $25 \mathrm{keV}$ is required for the electrons responsible for ECR absorption and emission processes.

Later in the discharge after the CFS formation ECE spectrum is still peaked with the maximum at $27.5 \mathrm{GHz}$ as indicated by blue triangles. Apparently plasma-wave interactions are shifted to a cooler fraction of electrons at this stage. After the end of RF injection in the constant or slowly ramping-up $\mathrm{Bv}$ the maximum of ECE is shifted back to $26.5 \mathrm{GHz}$ with enhanced emission at lower frequencies as shown in fig. 10b. That can be explained either by the presence of electrons with the energy of 25 $\mathrm{keV}$ and higher or by energetic electrons accumulated at larger radii where ECR frequency is lower due to the lower magnetic field. ECE signals measured at $N_{\|} \sim 1$ are typically twice stronger than at $N_{\|} \sim 0$ over the whole frequency range. The signals without RF injection are several times weaker compared with the signals measured in the presence of high power RF. There is a small ECE emitted from the inner radii with respect to the radius of a 'cold' ECR for the injected RF power. From these observations one can conclude that the observed spectra are of a cyclotron type in the contrast to a synchrotron type characterised by large relativistic broadening of ECR. Thus there are no relativistic electrons generated in the plasma during EBW start-up.

For comparison in fig. 10c are shown ECE spectra measured during RF injection with a limited solenoid ramp. The solenoid produced a loop voltage of $0.2 \mathrm{~V}$ for only $20 \mathrm{~ms}$ which was sufficient for generation of high energy electrons. The measured spectra are broad and of high intensity. They don't show any peaking and there is a big difference between $N_{\|} \sim 1$ and $N_{\|} \sim 0$ measurements.

\section{Conclusions}

Improvements, upgrades and associated testing of the high power $28 \mathrm{GHz}$ generation and transmission system used for the MAST EBW start-up, and current drive study have been performed in the past few years as a collaborative research between ORNL and CCFE. RF power injected into the vessel was of the same level as in previous experiments [1]. It was limited primarily by arcing at the vacuum window but the RF pulse length was increased by factor of 5 (up to $\sim 0.5 \mathrm{~s}$ ). Long pulse EBW start-up experiments on MAST have exceeded more than twice previously achieved plasma current levels. Closed flux surfaces (CFS) were clearly observed on fast video indicating that a tokamak like equilibrium was established during the EBW start-up phase. Plasma current shows an anti-phase response to the vertical modulation of the plasma midplane. That result proves that the current was driven by EBWs. The power scan indicates that the generated plasma current after CFS formation depends linearly on the RF power injected.
ECE measurements during EBW start-up support the hypothesis that plasma current is predominantly carried by supra-thermal electrons. No relativistic electrons are generated in the EBW assisted plasma start-up.

\section{Acknowledgements}

This work was part-funded by the RCUK Energy Programme under grant EP/I501045 and the European Communities under the contract of Association between EURATOM and CCFE. The views and opinions expressed herein do not necessarily reflect those of the European Commission.

\section{References}

1. V. Shevchenko et al, Nucl. Fusion 50, 022004 (2010)

2. C.B. Forest et al, Phys. Rev. Lett. 683559 (1992)

3. R. Raman et al, Phys. Rev. Lett. 90, 075005 (2003)

4. A. Ejiri et al, Nucl. Fusion 46, 709 (2006)

5. T. Maekawa et al, Nucl. Fusion 52, 083008 (2012)

6. B. Lloyd et al, NBI, Nucl. Fusion 43, 1665 (2003)

7. V. Shevchenko et al., Proc. 13th Joint Workshop on ECE and ECRH, Nizhny Novgorod, Russia 255, (2004)

8. V. Shevchenko et al, Fusion Sci. Technol. 52, 202 (2007)

9. V. Shevchenko et al, Phys. Rev. Lett. 89, 265005, (2002)

10. F. Jobes et al, Phys. Rev Lett. 52, 12, 1005, (1984)

11. C.B. Forest et al, Phys. Plasmas, 7, 5, 1352, (2000) 